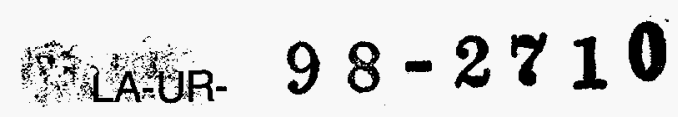

Approved for public release; distribution is unlimited.

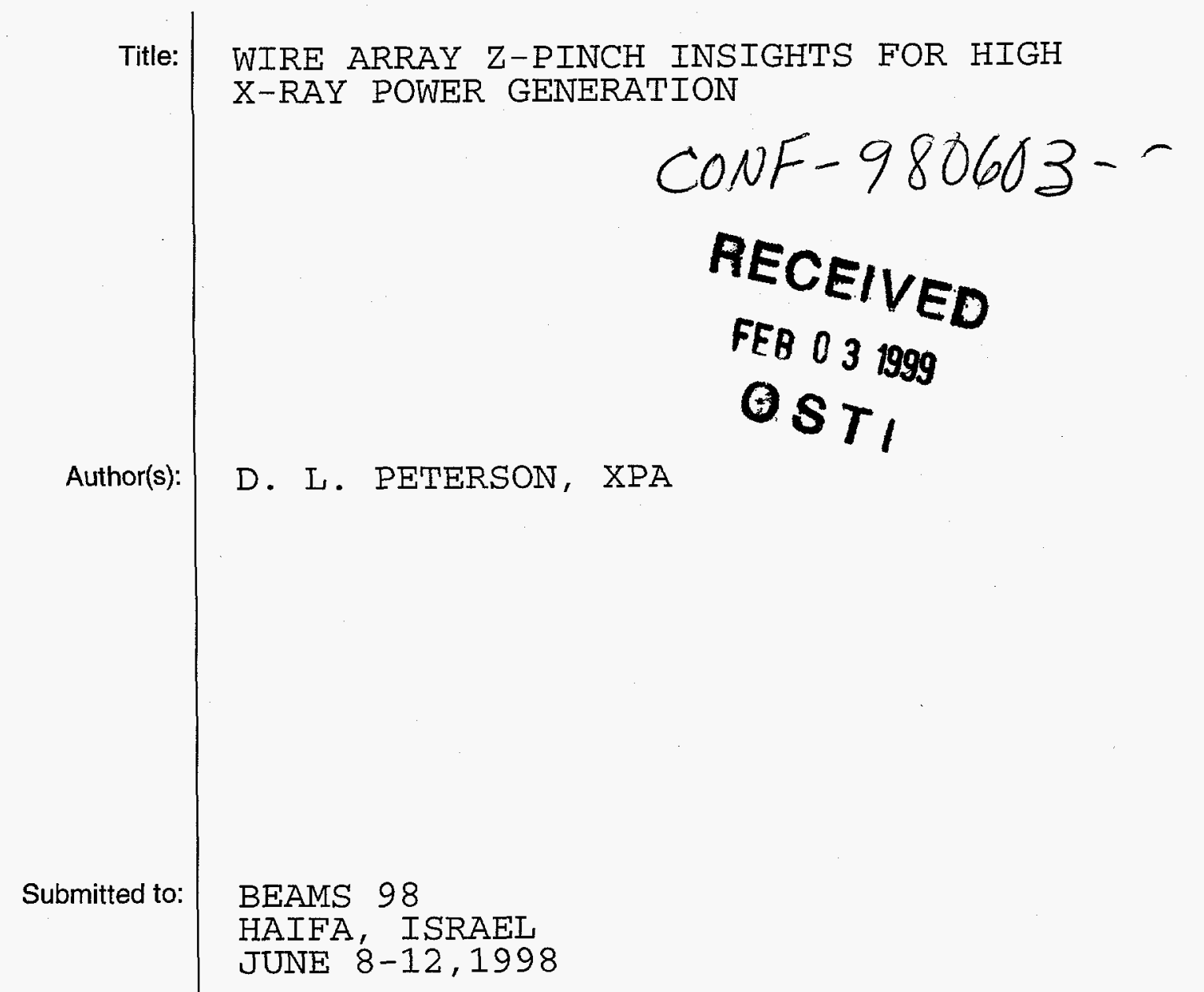

\section{Los Alamos}

NATIONALLABORATORY

Los Alamos National Laboratory, an affirmative action/equal opportunity employer, is operated by the University of California for the U.S. Department of Energy under contract W-7405-ENG-36. By acceptance of this article, the publisher recognizes that the U.S. Government retains a nonexclusive, royalty-free license to publish or reproduce the published form of this contribution, or to allow others to do so, for U.S. Govemment purposes. Los Alamos National Laboratory requests that the publisher identify this article as work performed under the auspices of the U.S. Department of Energy. The Los Alamos National Laboratory strongly supports academic freedom and a researcher's right to publish; as an institution, however, the Laboratory does not endorse the viewpoint of a publication or guarantee its technical correctness. 


\section{DISCLAIMER}

This report was prepared as an account of work sponsored by an agency of the United States Government. Neither the United States Government nor any agency thereof, nor any of their exployees, makes any warranty, express or implied, or assumes any legal liability or responsibility for the accuracy, completeness, or usefulness of any information, apparatus, produch, or process dicelosed, or represents that its use would not infringe privately owned rights. Referesce berein to any specific commercial product, process, or service by trade name, trademart, inanufac. turer, or otherwise does not necessarily constitute or imply its endorsement, recommendation, or favoring by the United States Government or any agency thereof. The views and opinions of authors expressed herein do not necessarily state or reflect those of the United States Governmeat or any agency thereof. 


\section{DISCLAIMER}

Portions of this document may be illegible in electronic image products. Images are produced from the best available original document. 


\title{
WIRE ARRAY Z-PINCH INSIGHTS FOR HIGH X-RAY POWER GENERATION*
}

\author{
T. W. L. Sanford, B. M. Marder, M. P. Desjarlais, R. C. Mock, T. J. Nash, R. B. \\ Spielman, \\ Sandia National Laboratories, P. O. Box 5800, Albuquerque,NM 87185 USA \\ D. L. Peterson \\ Los Alamos National Laboratory, Los Alamos, NM 87545; \\ K. G. Whitney, J. P. Apruzese, and P. E. Pulsifer \\ Naval Research Laboratory, Radiation Hydrodynamics Branch, Washington,DC 20375 USA \\ D. Mosher \\ Naval Research Laboratory, Pulsed Power Physics Branch, Washington, DC 20375 USA
}

\begin{abstract}
Comparisons of detailed measured implosion characteristics of annular wire array z-pinches with those modeled and simulated give insight into pinch dynamics and $\mathrm{x}$-ray power generation.
\end{abstract}

\section{INTRODUCTION}

The discovery [1] that the use of very large numbers of wires enables high $\mathrm{x}$-ray power to be generated from wire-array z-pinches represents a breakthrough in load design for large pulsed power generators, and has permitted high temperatures to be generated in radiation cavities [2,3] on Saturn [4] and $Z$ [5]. In this paper, changes in $x$-ray emission characteristics as a function of wire number, array mass, and load radius, for 20 -mm-long aluminum arrays on Saturn that led to these breakthrough hohlraum results, are discussed and compared with a few related emission characteristics of high-wire-number aluminum and tungsten arrays on $\mathrm{Z}$. $\mathrm{X}$-ray measurement comparisons with analytic models and 2-D radiationmagnetohydrodynamic (RMHC) code simulations in the $x-y$ [6] and r-z [7] planes provide confidence in the ability of the models and codes to predict future $\mathrm{x}$-ray performance with very-large-number wire arrays.

\section{RESULTS AND DISCUSSION}

Wire Number Variation: In the first set of Saturn aluminum wire-number experiments [1], the array mass was fixed and the wire number was varied by more than an order-of magnitude from 10 to almost 200 by simultaneously changing the interwire gap and the wire size. This procedure was carried out for a $0.62-\mathrm{mg}$ and a $0.84-\mathrm{mg}$ array having an initial radius of $8.6 \mathrm{~mm}$ and $12 \mathrm{~mm}$, respectively. The variation permitted interwire gaps to be explored from $6 \mathrm{~mm}$ down to $0.4 \mathrm{~mm}$ for both the small- and large-radius arrays. Decreasing the interwire gap resulted in monotonic decreases in the risetime and width of the $\mathrm{x}$-ray pulse and simultaneous increases in radiated power and energy, in the same way for both array radii (Fig. 1). Over the 6 to $0.4 \mathrm{~mm}$ gap reduction explored, the total radiated power increased by a factor of 20 (Fig. 1A) and the total radiated energy by a factor of 2 (Fig. 1C).

In addition, for gaps smaller than $2 \pm 0.6 \mathrm{~mm}$, the character of the $\mathrm{x}$-ray emission qualitatively changed, transitioning from a broad, single, irregular radiation pulse at large gaps, to a strong, narrow, evenly-shaped radiation pulse, that was followed by a much weaker pulse at small gaps (Fig. 2). The weaker pulse is consistent with a second radial implosion [8]. For gaps greater than $\sim 2 \mathrm{~mm}$, time-integrated images of the pinch exhibit the presence of a kink instability; time-dependent images show significant precursor plasma stagnating on axis, 
generating soft $\mathrm{x}$-ray emission tens of nanoseconds prior to the main implosion, in agreement with earlier experiments. For gaps less than $\sim 2 \mathrm{~mm}$, on the other hand, no kink instability is observed, with only a minimal precursor plasma forming. Moreover, the change in the temporal shape of the x-ray pulse (Fig. 2) and spatial quality of the pinch occurred along with corresponding quantitative transitions in the rates of change as a function of gap of (1) the emitted x-ray power (Fig.1A) and (2) energy (Fig. 1C), (3) the average size of the K-shell emission region (Fig. 1B), and (4) the average K-shell emitting ion density (Fig. 1D) and (5) 

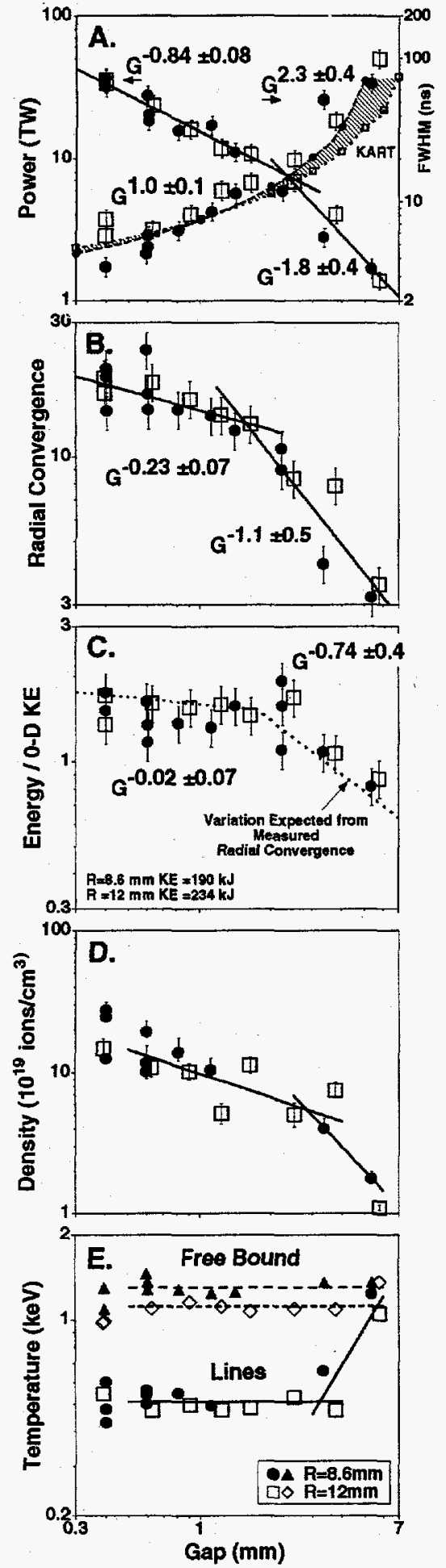

Fig. 1 (A) Peak total radiated power and pulsewidth and KART model, (B) Radial convergence, $(C)$ Total radiated energy normalized by calculated kinetic energy assuming a 10:1 convergence, (D) ion number density (K-shell region), and (E) electron temperature (K-shell region) versus gap. electron temperature (Fig. 1E). The emitting ion densities and electron temperatures were inferred from $\mathrm{x}$ ray size data together with the $\mathrm{K}$-shell power and $\mathrm{K}$-series spectrum data. Not enough data was taken at wide gap spacings, however, to ascertain how rapidly this transition in $\mathrm{x}$-ray behavior took place. For this reason, we represent it experimentally as a transition between two power laws as illustrated in Fig. 1, with the power indicated by the dependence on gap (G) shown.

Measurement of the slope of the optically-thin, free-to-bound, x-ray emission (Fig. 1E) determines the electron temperature of the hot core of the pinch [8]. It exhibits no variation with gap (dashed lines) and is only a function of the implosion kinematics. For large gaps, where the measured ion density is low, the temperature extracted from K-shell line ratios [9] (solid lines) agrees with that extracted from the free-to-bound emission. As the gap decreases and the emitting ion density increases (Fig. 1D), however, the optical depth of the K-shell emission becomes significant, and the line ratio begins to reflect the temperature of the outside surface of the emitting region, rather than an average over the region. This transition from a thin to a thick plasma, as indicated by the data of Fig. 1E, approximately coincides with the transition in the rate of change of density with gap (Fig. 1D) and with the transition at $\sim 2 \mathrm{~mm}$.

The difference between the core and surface temperature is indicative of a substantial temperature gradient within the emitting plasma. The enhanced plasma density at small gaps increases the temperature and density gradients and opacity effects, but in such a way as to approximately maintain the average amount of mass participating in the $\mathrm{K}$-shell emission at $\sim 11 \%$, independent of gap. Comparisons of the measured $\mathrm{K}$ shell emission radii with that simulated by the EulearianRMHC (E-RMHC) [7] indicate that the actual mass averaged radius is about double that extracted from the emission images [10]. This gradient structure is illustrated by a detailed analysis [11] of the x-ray image, spectral, and $\mathrm{K}$-shell power data, for an aluminum-array shot taken on $Z$, having an interwire gap of $0.5 \mathrm{~mm}$, a total mass of $4.1 \mathrm{mg}$, and an array radius of $20 \mathrm{~mm}$. The density and temperature profiles obtained from a best fit of a aluminum-plasma collisional-radiative-equilibrum model to these measured quantities is shown in Fig. 3. In the model, the radiative transfer is carried out for all optically-thick K-shell lines and two K-shell continuua temperatures. 
Interpretation of Number Variation: In general, variations in peak total power track the inverse of the measured pulsewidths (Fig. 1A), as would be expected if the total energy radiated during stagnation is slowly varying. The greater rate of dependence with gap of the pulsewidth relative to the peak power, for gaps greater than $\sim 2 \mathrm{~mm}$ reflects the greater disorganization of the implosion as seen by the lost double-pulse nature of the stagnation (Fig. 2). The accelerated rate of decrease in power for large gaps relative to that for small gaps is

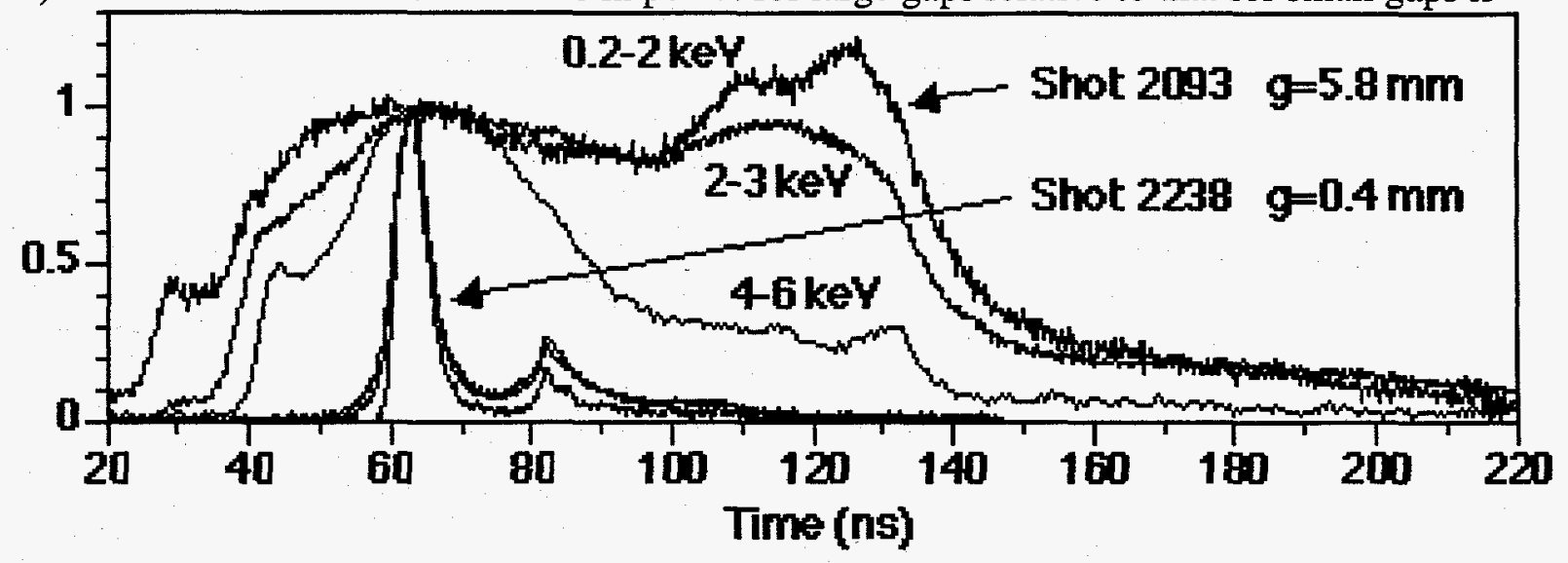

Fig. 2 Normalized pulseshape in three energy channels for two gaps.

consistent with the decrease in total radiated energy (Figs. 1A, 1C, and 4). This change in energy is approximately consistent with the change in the calculated (dotted lines in Fig. 1C) kinetic energy, and by inference, the radiated energy using the measured radial compression from the K-shell emission images (Fig. 1B). The trends in ion density (Fig. 1D) and electron temperature (Fig. 1E) demonstrate that the increase in power is the result of systematically greater plasma compression.

The apparent transition in implosion quality near $2 \mathrm{~mm}$ has been interpreted using a RMHC in $x-y$ geometry $[1,6]$. Calculations performed with this code show (in correspondence with the experimental data) that a change in the implosion topology occurs with increasing wire number. The implosion is seen to transition from one composed of non-merging, selfpinching individual wire plasmas to one characterized by the early formation and subsequent implosion of a quasi-plasma-shell. The shell had density and current variations distributed azimuthally that were correlated with the initial wire location and which decreased in amplitude with decreasing gap. The calculated transition region was sensitive to (1) the magnitude of the prepulse that accompanies the main current pulse, (2) the current flowing per wire, (3) the wire size, (4) the interwire gap, and (5) the resistivity model used. For the particular resistivity model used and for the measured prepulse and wire sizes used, this transition was found to occur between wire numbers 20 to 40 (or between interwire gaps of about 3 to $1 \mathrm{~mm}$, respectively), for the small radius load [1]. This calculated transition was also seen to be consistent with observations made with $1.3-\mathrm{mm}$ gap loads in the transition region. There, individual wires were observed to self-pinch $20 \mathrm{~ns}$ prior to peak radiated power (where the array had only imploded a fraction of a $\mathrm{mm}$ radially). Ten nanoseconds later, after the array had imploded an additional $1.5 \mathrm{~mm}$, the observed plasma emission became a continuous distribution, with no evidence of individual wire structure. 


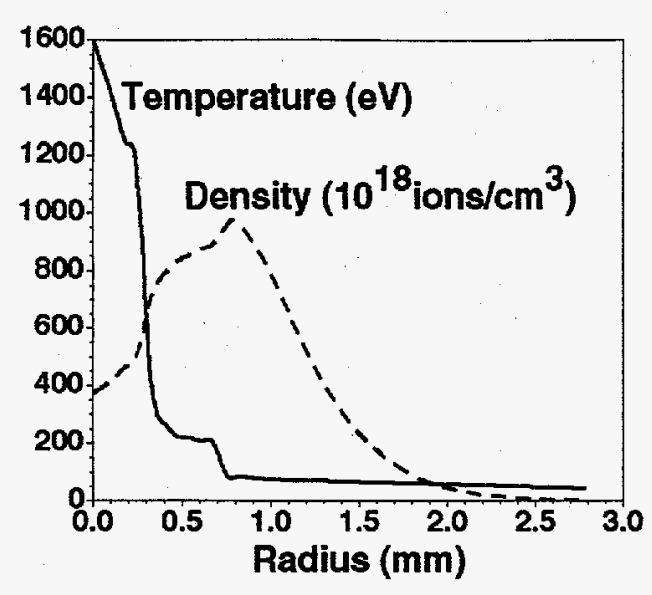

Fig. 3 Calculated ion density and electron temperature for aluminum $Z$ shot.

The x-y simulations [6] together with analytic modeling [12] show that the wire-plasmas, in contrast to the plasma of a shell, accrue azimuthal velocity components during the implosion owing to deviations in the locations of the individual wires from those of a perfect annulus or to the presence of the limited number

of current return posts surrounding the array [12] These velocity components produce density asymmetries at stagnation that reduce both the compressibility of the stagnating plasma and the resulting radiated energy, both in qualitative agreement with the discontinuity observed in the radial convergence measurements (Fig. 1B) and energy channel (Fig. 1C). The $x-y$ simulations show, however, that these variations cannot account for the change in measured pulse shape, for any wire number greater than 10. In contrast, E-RMHC [7] simulations

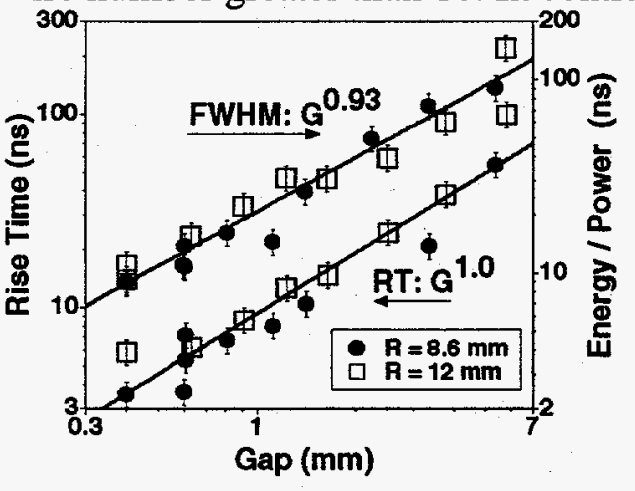

Fig. 4 Risetime and effective pulsewidth versus gap.

in the $\mathrm{r}-\mathrm{z}$ plane, which assume an azimuthally symmetric plasma shell with a random density distribution in the r-z direction, suggest that the shape of the primary power pulse and the general change in peak power with gap are related to the evolution of the thickness of the plasma sheath due to r-z motions and the growth of Rayleigh-Taylor (RT) instabilities. This thickness is calculated to scale linearly with the pulse width. The measured risetime of the total radiation pulse and the "effective" pulsewidth (defined as the total energy divided by the peak power) scale as the gap, over the entire gap range explored, showing no discontinuity near $2 \mathrm{~mm}$ (Fig. 4). This data, together with the simulations, thus suggest a direct relation between the initial interwire gap and the resulting thickness of the imploding sheath.

Recently, an analytic model (KART) of the implosion has been developed by Desjarlais and Marder [13] that takes into account an amplification of the RT instability arising from a kink instability, which deforms the individual wire plasmas. This deformation is in phase with that generated by the RT instability, which is assumed to arise from a global sausage instability acting on the entire array. The agreement of their estimated pulsewidth with that measured for these experiments (dashed curves in Fig. 1A), as well as with that measured for other tungsten experiments on Saturn [14] and Z [5, 15], with only one perturbation parameter that scales with the wire size, suggests that the wires retain enough of their individual identity for a sufficient time to allow the kink amplification to participate. Doubling the number of tungsten wires on $\mathrm{Z}$ from 120 to 240 , for example, reduced the measured pulsewidth by $(29 \pm 9) \%$, in agreement with a calculated $25 \%$ reduction.

Mass and Radius Variation: Two sets of additional Saturn aluminum-wire experiments were conducted in the calculated high-wire-number quasi-plasma-shell regime [10]. These experiments show two important trends. Firstly, when the mass of the 12-mmdiameter arrays is reduced from above 1.9 to below $1.3 \mathrm{mg}$, a factor of two decrease in pulsewidth (Fig. 5) occurs for all ten energy channels measured between 0 and $10 \mathrm{keV}$. The 
associated peak power in the energy channels exhibits a two to twenty fold increase, respectively. Secondly, when the radius is increased from 8.6 to $20 \mathrm{~mm}$, for a mass of $0.6 \mathrm{mg}$, the experiments show that the radiated pulsewidth (Fig. 6) in all channels increases from only $\sim 4$ to $\sim 7 \mathrm{~ns}$. The associated peak powers in all channels remain relatively unchanged with radius.

Interpretation of Mass and Radius Variation: The E-RMHC simulations were used to understand the underlying pinch dynamics. Over the mass range of 0.42 to $3.4 \mathrm{mg}$ and radius range 8.6 to $20 \mathrm{~mm}$ measured, which spanned an implosion time of 40 to $90 \mathrm{~ns}$, the

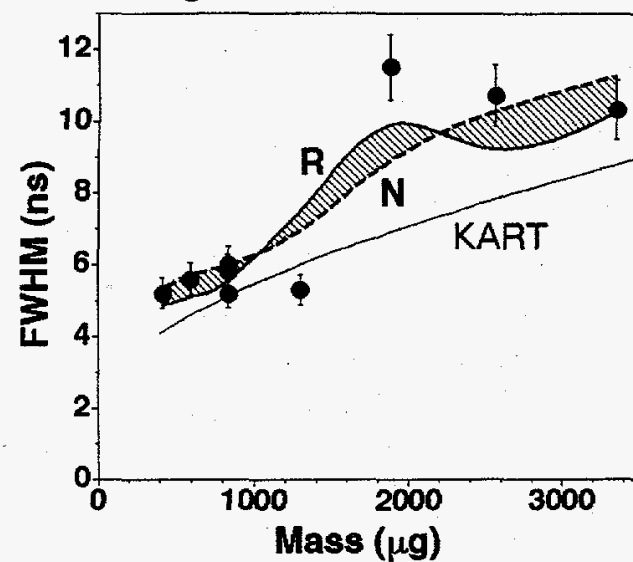

Fig. 5 Measured, modeled, and simulated pulsewidth versus mass. implosion time of the simulated pulse agrees with that measured within a shot-to-shot variation of only $2 \mathrm{~ns}$. This agreement suggests that $(100 \pm 6) \%$ of the initial mass is being accelerated during the implosion. For these simulations, the electron-photon coupling was set to either its nominal value (indicated by $\mathrm{N}$ ) or a reduced value (indicated by $R$ ), such that the calculated peak total radiated power agreed with that measured at $\sim 0.6 \mathrm{mg}$. Within the uncertainty of this emissivity approximation, the measured pulsewidth (Figs. 5 and 6) and trends in total radiated peak power agree with that simulated, using only a single value of a density perturbation seed [10]. KART calculations are

also shown in Figs. 5 and 6.

For all cases, the E-RMHC simulations show a two-stage development of the instability with initial bubble burst when a wavelength, of the order of the

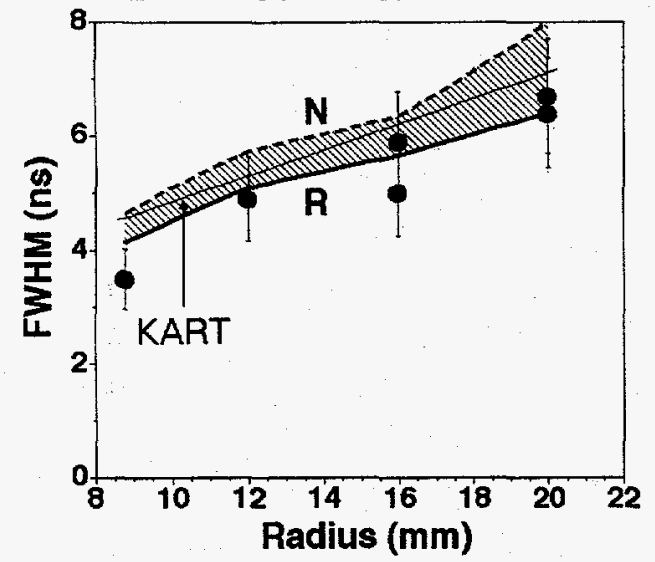

Fig. 6 Measured, modeled, and simulated pulsewidth versus radius. shell thickness, is reached, followed immediately by current flow in the low-density material, between the spikes. The plasma shell self heals and continues to accelerate with only a small amount of bubble material being thrown ahead of the main body of plasma. The instability growth continues, evolving to longer wavelengths, until one of the order of the new shell thickness is reached. When the shell bursts the second time (at longer wavelengths), a significant amount of material is accelerated to the axis, and the radiation pulse begins. These simulations show that the decrease in pulsewidth and associated doubling of the peak total power, as mass is reduced, is due to the faster implosion velocity of the plasma shell relative to the growth of the shell thickness. The relative increase in peak power for the higher-energy $x-$ rays is due to an increase in ion temperature, arising from an increase in kinetic energy per incident ion at stagnation. The simulations also show that the increase in pulsewidth with radius is due to the faster growth of the shell thickness relative to the increase in shell velocity. These results suggest that the improved uniformity provided by the large number of wires in the initial array reduces the disruptive effects of instabilities observed in small-wirenumber imploding loads. 
The simulations generate total radiation pulse shapes in agreement with the primary pulse measured, as illustrated in Fig. 7 for a $0.84-\mathrm{mg}, 12-\mathrm{mm}$ radius load. The simulations indicate that the energy deposited in the plasma arises primarily from the Lorentz $(\mathbf{J} \times \mathbf{B})$ force and goes primarily into accelerating the plasma, increasing its kinetic energy. At early times when instabilities have not become important and there has been little plasma heating, the simulated energy deposited by the Lorentz force and the plasma radial kinetic energies are nearly equal. At later times, the instability destroys the plasma shell, accelerating plasma to the axis where it stagnates, and the kinetic energy diverges from the work generated by the Lorentz force. The plasma that has not stagnated continues to be accelerated by the Lorentz force. Because the radiation rate is higher than the rate at which energy is being supplied, the total kinetic-energy decreases, even though some plasma continues to be accelerated. As the

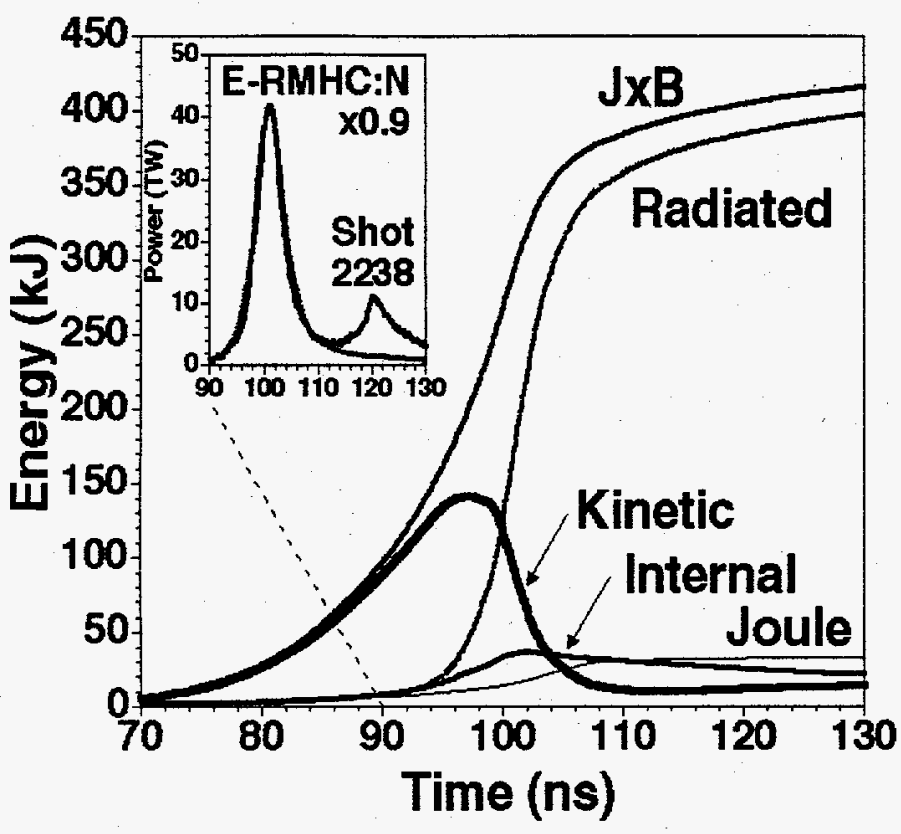

Fig. 7 E-RMHC simulated energy partition. pressure rises, some of the $(\mathbf{J} \times \mathbf{B})$ energy is transferred to internal energy by pdV work, rather than as a kinetic-energy increase. Due to the extended radial nature of the plasma as stagnation begins, the net result on the energy flow is that part of the plasma on axis releases energy as radiation, while regions away from the axis continue to absorb energy, which may then be radiated later in the pulse. The result is a total radiated energy that is higher than the instantaneous peak in the kinetic energy at the time stagnation begins. The calculations show only a small contribution from Joule heating.

The variation with mass on $Z$ using tungsten wires in the high-wirenumber regime shows similar trends to those observed on Saturn for the aluminum wires [5, 15]. On $\mathrm{Z}$, the pulsewidth decreased by a factor of two and the total radiated power doubled when the mass decreased from 6 to $4 \mathrm{mg}$, for loads having a 20-mm radius. Moreover, these high-wire-number implosions, with interwire gap of $0.5 \mathrm{~mm}$, produced high-quality implosions that had pulsewidths of only $7 \mathrm{~ns}$ at peak power.

A New Model: The results show that good agreement with radiation pulse widths derive from an initial perturbation that depends only on the interwire gap. Single-wire observations and computations in carbon and aluminum [16, 17] suggest a mechanism [18] as follows that may help to explain the dependence in contrast to KART. Uncorrolated wire-to-wire sausage modes in individual wire plasmas grow until the plasmas of adjacent wires merge, after which current flow in the plasma shell stops their growth. At the merging time, the thickness of the annulus is then roughly the same as the initial gap. A key observation of the single-wire studies is that the sausage perturbations are nonlinear and large-amplitude even at very early times in their development, with a shape that is self-similar in the radial scale, that is, they ilookî about the same independent of radius. This structure is also observed in ongoing tungsten linear-wire-array experiments on Naval Research Laboratory Gamble II generator that simulate $\mathrm{Z}$ prepulse conditions. From the self-similar observation and analysis of the perturbation spectrum, the fundamental (longest) wavelength in the perturbation is also about 
the size of the interwire gap. Thus, in this model, the amplitude of the initial perturbation is fixed while its wavelength scales with gap.

E-RMHC computations are required to determine if such initial conditions can reproduce the observed dependence of radiation characteristics without an arbitrarily-chosen perturbation amplitude for a given gap. However, the self-similar model does provide for RT saturation and healing for sufficiently-short wavelength (small gap/large wire number). Also, existing E-RMHC computations do show that tripling the scale of initial $10 \%$ and $15 \%$ initial perturbations is equivalent to shorter-scale perturbations of $45 \%$ and $75 \%$, demonstrating that perturbation wavelength is a powerful determining factor for implosion quality and radiation pulse width.

\section{CONCLUSION}

Implosions that develop narrow pulsewidths with high peak powers can be generated from both small- and large-radius annular wire arrays by keeping the interwire gap to spacings on the order of $0.5 \mathrm{~mm}$ or less. Reducing the implosion time, while still providing good current coupling to the load at stagnation, reduces the growth of the radial instabilities relative to the implosion velocity and permits the highest powers to be developed. The RMHC simulations and the Desjarlais-Marder model agree with aspects of the data and provide insight into the underlying dynamics. A new self-similar model for the initial E-RMHC perturbation dependence on gap provides additional insight and may improve that codes predictive capability for future large-wire-number experiments on $\mathrm{Z}$.

[1] T. W. L. Sanford, et al, BEAMS'96, pp.146-149; Phys. Rev. Lett. 77,5063 (1996).

[2] M. K. Matzen, Phys. Plasmas 4, 1519 (1997).

[3] J. P. Quintenz, et al, this conference.

[4] D. D. Bloomquist, et al, Proc. 6th Int. IEEE Pulsed Power Conf., ( 1987), p. 310.

[5] R. B. Spielman, et al, BEAMS'96, pp 150-153; Phys. Plasmas 5, 2105 (1998).

[6] B. M. Marder, et al, to be published Phys. Plasmas (1998).

[7] D. L. Peterson,et al, Phys. Plasmas 3, 368 (1996).

[8] T. W. L. Sanford, et al, Phys. Plasmas 4, 2188 (1997).

[9] K. G. Whitney, et al, Phys. Rev. E 56, 3540, (1997).

[10] T. W. L. Sanford, et al, Dense Z-Pinches 4th Int. Conf. AIP (1997), pp 561-573.

[11] J. P. Apruzese, et al, to be submitted to Phys. Plasmas (1998).

[12] D. Mosher, BEAMS'94, pp 159-162.

[13] M. P. Desjarlais and B. M. Marder to be submitted to Phys. Plasmas (1998).

[14] C. Deeney, et al, Phy. Rev. E 56, 5945 (1997).

[15] T. W. L. Sanford, et al, to be published IEEE Trans. Plasma Sci. (1998).

[16] F.N. Beg, et al., Plasma Phys. And Controlled Fusion, 39, 1-25(1997).

[17] J.P. Chittenden, et al., Bull. Am. Phys. Soc. 41, 1471(1996).

[18] D. Mosher, et al.,"Modeling Initial Annular Perturbations in Large Wire-Number Arrays",work in progress.

*Sandia is a multiprogram laboratory operated by the Sandia Corp., a Lockheed Martin Co., for the US DOE under Contract DE-AC04-94AL85000. 